\title{
A case of syringomyelia in a 12 year old child
}

P M G Punchihewa ${ }^{1}$, M C G Karunanayake ${ }^{2}$

Sri Lanka Journal of Child Health, 2002; 31: 23-4

(Key words: syringomyelia, child)

\section{Introduction}

Syringomyelia is defined as a chronic, progressive degenerative disorder of the spinal cord characterised by dissociated sensory loss and brachial amyotrophy with pathological evidence of central cord cavitation ${ }^{1}$. When this cavity extends into the medulla, it is called syringobulbia ${ }^{1}$. The expanding cavity gradually destroys the second order neurones of spinothalamic tracts, anterior nerve cells and lateral corticospinal tracts ${ }^{1}$.

Pathogenesis of this disease is unclear. The prevailing hypothesis suggests a constriction of the central canal at the level of the foramen magnum during embryogenesis. Cerebrospinal fluid (CSF) may pass caudal to the narrowed central canal and produce dilatation of the canal ${ }^{2}$.

Barnett et $\mathrm{al}^{3}$ proposed the following broad classification:

1. Communicating syringomyelia (also called "syringohydromyelia").

2. Non-communicating syringomyelia.

The fundamental distinction between these 2 groups of disorders is the presence or absence of a communication between the cord cavity and the CSF pathways.

Communicating syringomyelia is frequently associated with the Chiari type 1 malformation whereas non-communicating syringomyelia is associated with cord tumours, vascular accidents and trauma ${ }^{4}$.

Because of its slow evolution, syringomyelia rarely produces symptoms during childhood ${ }^{5}$.

${ }^{1}$ Consultant Paediatrician, ${ }^{2}$ Paediatric Registrar, Teaching Hospital, Karapitiya.

\section{Case report}

A 12 year old school girl, an athlete, presented for evaluation of a mild headache of 3 years duration.

Though an athlete, she has not experienced any significant trauma to her cervical spine. Examination revealed a child with bilateral exophthalmos and a mild torticollis to her right. The only neurological abnormality detected was the presence of exaggerated tendon reflexes in the lower limb with a negative Babinski response.

Laboratory evaluation revealed normal full blood count, erythrocyte sedimentation rate, normal thyroid function tests and a negative rheumatoid factor. Xray of the cervical spine (open mouth view) did not reveal any abnormality. Computed tomography (CT) of the brain was normal.

Since the investigations did not reveal any positive findings and as her headache responded to analgesics, she was closely followed up in the clinic. Over the next few months her headache became worse and she started to complain of dysphagia and deterioration of handwriting.

On examination at this stage, she was found to be unsteady and dysarthric. There was mild wasting of the small muscles of both hands with early pes cavus deformity. Her lower limb reflexes remained exaggerated with extensor plantar response. There was mild sensory loss in the distal extremities of the upper limb to pain and temperature without any evidence of a sensory level, whilst the sense of simple touch and proprioception were spared. There was no sphincter involvement and the cranial nerves were normal.

Magnetic resonance imaging (MRI) of the cervical spine showed a syrinx which was extending to the brain stem. Arnold Chiari malformation was not seen (Figure 1). 


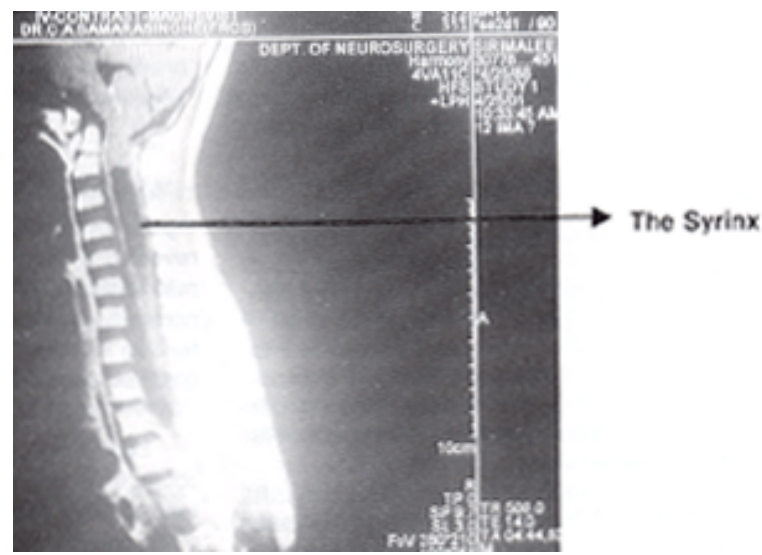

Figure 1 MRI scan demonstrating the syrinx

She underwent craniovertebral decompression surgery along with cervical laminectomy. At present, apart from persistent torticollis and mild wasting of the small muscles of the hands, she is asymptomatic.

\section{Discussion}

Syringomyelia is a rare disorder to have presented at 12 years of age ${ }^{5}$. Due to its slow evolution patients usually present in 3rd or 4th decade of life.

Cavitation around or near the central canal of the cervical spine interrupts the lateral spinothalamic fibres as they cross from one side to the other, resulting in loss of pain and temperature sense in the upper limbs. But the proprioception and the sense of simple touch were spared in the upper limb. This sensory dissociation is most characteristic of syringomyelia. Since the spinothalamic tracts themselves remain intact there was no sensory impairment in the lower limbs.

Wasting of the small muscles of the hands in our patient was secondary to extension of the lesion to anterior gray horns. The upper motor neurone signs of the lower limbs was a result of progressive compression of the pyramidal tracts. Dysphagia and dysarthria were caused by the presence of syringobulbia.
When she presented to us initially with headache, the positive physical signs were exophthalmos (later revealed to be congenital), torticollis and exaggerated lower limb reflexes with flexor plantar reflexes. As the neurological signs were very subtle, once we excluded cervical spine abnormality and slow growing brain lesion, we did not pursue with a MRI scan which would have given us an early diagnosis.

Insight into the neuroanatomical location became more obvious when she developed additional physical signs making us consider the MRI scan which is the investigation of choice.

Surgical decompression around the foramen magnum or syrinx itself is the treatment ${ }^{4}$. It will arrest the progression of neurological signs and often relieves pain. However it is often not successful and the condition tends to progress or worsen if brain stem is involved ${ }^{4}$.

\section{References}

1. Adams R D, Victor M. Principles of Neurology. 3rd ed. New York; Mcgraw-Hill, 1985: 665-698.

2. Newman P K, Terenty T R, Foster J B. Some observations on the pathogenesis of syringomyelia. J Neurol Neurosurg Psychiatry 1981; 44: 964.

3. Barnett J H M, Foster J B, Hudgson P. Syringohydromyelia. London; Saunders, 1973.

4. Cahan L D, Bentson J R. Considerations in the diagnosis and treatment of syringomyelia and Chiari malformation. J Neurosurg 1982; 57: 24.

5. Zager E I, Ojemann R G, Poletti C E. Acute presentations of syringomyelia: Report of three cases. J Neurosurg 1990; 72: 133-8. 
